\title{
Tipología de la empresa innovadora en productos y procesos en una región periférica europea
}

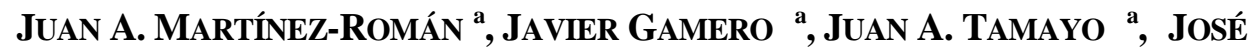 \\ E. ROMERO ${ }^{\text {a }}$ \\ a Universidad de Sevilla, Facultad de CC.EE. y EE., Avda. Ramón y Cajal, s/n, 41018 Sevilla, \\ España.E-mail: jamroman@us.es, jgam@us.es, jtamayo@us.es, romerogje@us.es
}

\begin{abstract}
RESUMEN
El gap de conocimiento sobre el proceso de innovación en regiones económica y tecnológicamente menos desarrolladas o periféricas ha perjudicado el impacto económico esperado de las medidas de fomento innovador en estas regiones. El objetivo principal del presente trabajo es caracterizar a la empresa innovadora respecto a la empresa que no innova en Andalucía, una importante región española y una de las principales regiones periféricas de la Unión Europea. Para el trabajo empírico se ha utilizado una encuesta realizada a 430 empresas andaluzas de cuatro sectores productivos, tecnológicos y no tecnológicos, distinguiendo entre la innovación en productos y la innovación en procesos. El análisis de la tipología revela rasgos comunes asociados al tipo de innovación. Así, la organización que innova en productos se caracteriza por el desarrollo de una capacidad innovadora donde las fuentes de conocimiento interno predominan sobre las fuentes externas. Por su parte, la organización que innova en procesos fundamenta su capacidad innovadora en el conocimiento interno y en ciertas fuentes externas.
\end{abstract}

Palabras clave: Innovación, empresa innovadora, región periférica, PYME, Andalucía.

\section{Typology of Firms Innovating in Products and Processes in a European Peripheral Region}

\begin{abstract}
The knowledge gap on the innovative process in economically and technologically less developed or peripheral regions has harmed the expected economic impact of innovative measures in these regions. The main objective of this paper is to characterise innovative firms in contrast with non-innovating companies in Andalusia, an important Spanish region and one of the main peripheral regions of the European Union. For the empirical research, a survey on 430 Andalusian firms from four technological and non-technological sectors was carried out, distinguishing between product innovation and process innovation. The analysis of the typology reveals common features associated with each type of innovation. Thus, the organization that innovates in products is characterised by the development of an innovative capacity where the sources of internal knowledge predominate over external sources. On the other hand, the organization that innovates in processes bases its innovative capacity on internal knowledge and on certain external sources.
\end{abstract}

Keywords: Innovation, Innovative Firm, Peripheral Region, SME, Andalusia.

\section{Clasificación JEL: M20, O31, O32, C81}

\footnotetext{
* Este trabajo ha utilizado la base de datos del proyecto PRY096/11, financiado por la Fundación Pública Andaluza Centro de Estudios Andaluces (Departamento de Presidencia de la Junta de Andalucía, España) en el marco de la VII edición de Proyectos de Investigación. Los autores también expresan su gratitud a los editores y revisores por sus comentarios y sugerencias.
}

Artículo recibido en mayo de 2017 y aceptado en agosto de 2017

Artículo disponible en versión electrónica en la página www.revista-eea.net, ref. ə-35306 


\section{INTRODUCCIÓN}

La innovación es un proceso complejo encaminado a la introducción de novedades en el mercado que ha demostrado tener una notable influencia en el crecimiento económico, el empleo y la competitividad de las economías (Dosi, Pavitt y Soete, 1990; Freeman, 1987; Porter, 1991). Así, la innovación se ha convertido en objetivo preferente de su actuación política y eje vertebrador del desarrollo económico en la Unión Europea. Los diversos programas comunitarios, nacionales y regionales, buscan por diversas vías el fomento de la actividad innovadora de las empresas para facilitar el tránsito a un nuevo modelo de desarrollo económico basado en la innovación y la competitividad para Europa (European Commission, 2010).

La especificidad del proceso innovador y la relevancia de los factores del entorno en la innovación justifican que la investigación de este complejo fenómeno se realice en un ámbito geográfico lo más homogéneo posible. Así, diversos argumentos señalan al ámbito regional como el marco adecuado para el estudio empírico del proceso de creación, difusión y aplicación del conocimiento (Fritsch y Andreas, 2005; Kaufmann y Tödtling, 2001), dada la influencia de las características regionales en la actividad innovadora de las empresas (Rondé y Hussler, 2005). Diferentes estudios han señalado la existencia de "fallos" en la política de innovación regional en Europa, llegando a la conclusión de que no existen modelos de innovación generales, eficaces en cualquier contexto, y que las políticas de innovación deben adaptarse a las características específicas de las regiones (Asheim y Coenen, 2005; Tödtling y Trippl, 2005). El informe Regional Innovation Scoreboard (European Commission, 2009, 2012a) insiste en la necesidad de políticas que reflejen el contexto regional de la innovación para poder mejorar los resultados de las economías regionales europeas menos favorecidas a través del fomento de la innovación en las empresas, apostando claramente por una regionalización de la política de innovación en la comunidad (Fritsch y Andreas, 2005).

A pesar de que el entorno regional es un factor esencial del proceso de innovación a nivel micro y macro económico, existe un déficit de conocimiento del fenómeno en las regiones periféricas tecnológicamente más atrasadas. Tödtling and Trippl (2005) caracterizan a la región periférica por el predominio de pymes, el bajo nivel de actividades de investigación y desarrollo, una escasa orientación hacia la demanda, las debilidades del sistema de formación y entrenamiento, los fallos en la generación y difusión tecnológica, los insuficientes servicios especializados y una baja densidad de redes y clústeres de empresas. Otros autores señalan como rasgos propios de este tipo de regiones la baja presencia de sectores tecnológicos, la menor actividad innovadora y la mayor dependencia de las universidades en estas regiones (Bilbao-Osorio and 
Rodríguez-Pose, 2004), así como su menor gasto en R\&D y un menor número de patentes registradas (Fritsch, 2000).

La implantación de modelos de innovación de regiones desarrolladas en las regiones periféricas ha ocasionado fallos e ineficiencias de la política de innovación en éstas últimas. Así, por ejemplo, la tendencia a implantar modelos únicos o generales de innovación y el énfasis por injertar los proyectos de alta tecnología en estas economías ha llevado a financiar centros tecnológicos y de transferencia de conocimientos en regiones sin tradición investigadora ni suficiente iniciativa empresarial, normalmente con escasos resultados. El limitado efecto derrame (spillover) en estas regiones es un claro ejemplo de la desconexión existente entre la actividad de los centros tecnológicos y las necesidades reales de las SMEs. Kaufmann and Tödtling (2002) analizan las consecuencias de la insuficiente efectividad de las actividades de apoyo, detectando desajustes entre la ayuda requerida y ofrecida, señalando las consecuencias de apoyar a ciertos beneficiarios en detrimento de otras empresas locales que podrían obtener mejores resultados innovadores y económicos.

El enfoque microeconómico, o a nivel de empresa, puede ser una vía factible para superar las actuales limitaciones del análisis agregado en el ámbito regional y aumentar la eficacia de la política de innovación en regiones caracterizadas por una fuerte presencia de actividades no tecnológicas (Howells, 2005; Uyarra, 2010). Sin embargo, el limitado conocimiento que aún existe sobre la empresa innovadora (Romijn y Albaladejo, 2002; Martínez-Román, Gamero y Tamayo, 2011), especialmente en sectores menos tecnológicos (Martínez-Román, Tamayo, Gamero y Romero, 2015; Martínez-Román, Tamayo y Gamero, 2017), dificulta notablemente el objetivo final de las políticas e instituciones de soporte de la innovación a nivel regional, que no es otro que el aumento de la capacidad innovadora de las empresas locales (Kaufmann y Tödtling, 2002).

El presente trabajo se sitúa en esta línea de investigación, caracterizando a la empresa innovadora respecto a la empresa que no innova en una región periférica europea, tanto en sectores tecnológicos como no tecnológicos. De este modo, el objetivo del trabajo es identificar las claves del comportamiento innovador de las organizaciones en regiones menos desarrolladas o tecnológicamente menos avanzadas, en contraposición al resto de empresas del entorno, proporcionando hallazgos útiles para directivos y responsables de la política de innovación en este ámbito geográfico.

Para alcanzar dicho objetivo, el trabajo ha sido estructurado del siguiente modo: tras esta introducción, en la sección segunda se desarrolla el marco teórico y se propone un modelo conceptual para la elaboración de las tipologías de empresas innovadoras, en productos y procesos. Los detalles de la investigación empírica aparecen en la sección 3, mientras que los resultados del 
análisis figuran en la sección 4. Finalmente, la sección 5 contiene el apartado de discusión y conclusiones con los principales hallazgos del trabajo.

\section{MARCO TEÓRICO}

El enfoque micro-nivel o nivel empresa es un ámbito de estudio relevante en el análisis de la innovación a nivel regional (Uyarra, 2010). Esta vía de investigación, al centrar su atención en la actividad innovadora de las empresas, constituye un valioso instrumento para el diseño de políticas de innovación a nivel regional (Howells, 2005). Estos trabajos buscan patrones de innovación empresarial en contextos específicos, regionales, sectoriales o tecnológicos, elaborando modelos conceptuales donde se agrupan por categorías el conjunto de factores internos y externos que explican el resultado innovador de las organizaciones (e.g. Forsman, 2011; Hurley y Hult, 1998; Vega-Jurado et al., 2008; Yam, Lo, Tang y Lau, 2011). Estos modelos aportan una visión global del impacto de ambas categorías básicas de variables explicativas sobre el comportamiento innovador de las organizaciones y sus resultados.

Sin embargo, este tipo de estudios suelen dirigir su atención a los sectores más tecnológicos y a las regiones más avanzadas, ya que las áreas con mayor concentración de conocimiento tecnológico suelen obtener los mejores resultados innovadores, denominándose "acumulabilidad” o "ventajas acumuladas" a este fenómeno en la literatura (Howells, 2005; Chen y Guan, 2011). Esta situación ha llevado a enfatizar las actividades de I+D en la investigación empírica de la empresa innovadora, relegando el estudio de los sectores maduros, las actividades menos intensivas en tecnología y la capacidad innovadora de las empresas a un plano secundario en la investigación empírica y la política de innovación (Tödtling and Trippl, 2005; Martínez-Román et al., 2011).

Una revisión de la literatura ha permitido elaborar el modelo conceptual para el análisis micro-nivel de la innovación que se muestra en la Figura 1. Este modelo, que ha demostrado su validez estadística en sectores tecnológicos y no tecnológicos (Martínez-Román et al., 2011), explica el nivel de innovación en productos y procesos a partir de tres categorías de variables explicativas: la capacidad innovadora, los factores contextuales y el entorno de la empresa, con subcategorías que introducen en el análisis variables que han demostrado su relevancia en la literatura (Martínez-Román et al., 2011). El contraste empírico del modelo supone la validación estadística de las siguientes hipótesis:

H1. Los resultados innovadores en productos pueden expresarse con un modelo lineal generalizado de variables explicativas referidas a la capacidad innovadora, los factores contextuales y el entorno.

H2. Los resultados innovadores en procesos pueden expresarse con un modelo lineal generalizado de variables explicativas referidas a la capacidad innovadora, los factores contextuales y el entorno. 
A partir del contraste del modelo representado en la Figura 1 se obtendrán las variables significativas que mejor explican el nivel de innovación en productos y procesos de las empresas de la región, tanto en sectores tecnológicos como no tecnológicos. Este conjunto de variables relevantes será utilizado en la presente investigación para caracterizar a las empresas innovadoras frente a las que no innovan, tanto en productos como en procesos, y extraer las consecuencias oportunas para los responsables políticos y los directivos de empresas en el contexto regional analizado. De este modo el trabajo supondrá un avance para el análisis micro-nivel de la innovación en regiones periféricas europeas.

Figura 1

Modelo conceptual

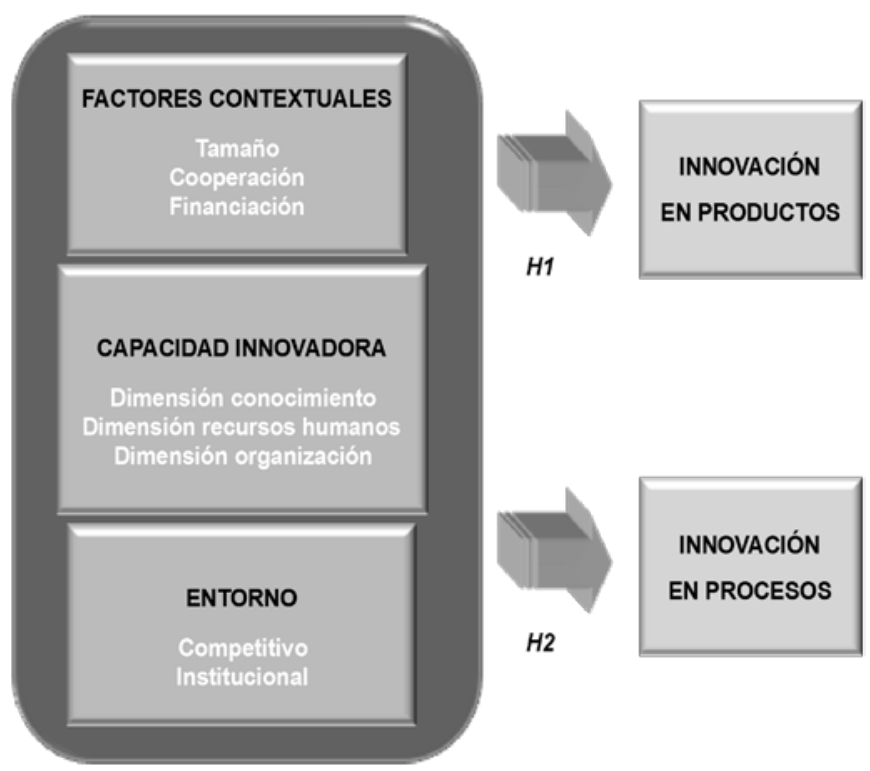

Fuente: Elaboración propia a partir de Martínez-Román, Gamero y Tamayo (2011).

\section{INVESTIGACIÓN EMPÍRICA}

\subsection{Datos}

Andalucía (NUTS 2 ES61) es una región española situada en el sur de la Península Ibérica que se caracteriza por su bajo nivel de desarrollo económico y tecnológico en la Unión Europea (European Commission, 2012b). Con una población de 8.2 millones de habitantes, Andalucía es la tercera región por número de habitantes de E.U. y la más poblada entre las regiones perceptoras de fondos de cohesión europeos. La renta per cápita andaluza es de 17,405 €, lo que supone el $75.5 \%$ de la media nacional y el $71.1 \%$ de la media UE-27, y sólo 
el 62\% de la media de los países de la Eurozona, mientras que su tasa de desempleo es del $27,97 \%$, un $8 \%$ mayor que la media española y el $18 \%$ mayor que la media de UE-27, según datos de 2010 de Eurostat.

Andalucía pertenece al grupo de 52 regiones europeas menos innovadoras del último informe europeo The Regional Innovation Scoreboard, lo que supone un retroceso respecto a su clasificación en 2009 (European Commission, 2012a), siendo el gasto en I+D respecto al PIB la mitad de la tasa registrada en la Zona Euro (European Commission, 2012a), generándose la mayor parte de este gasto en instituciones y organismos públicos (European Commission, 2012b). Por tanto, Andalucía puede considerarse como una región representativa de los territorios periféricos europeos para el estudio de la innovación empresarial.

\subsection{Muestra}

La selección de la muestra ha sido realizada mediante una variedad de muestreo aleatorio corregido por estratos para maximizar el análisis por sectores y manteniendo unas cuotas mínimas por provincia. Los sectores considerados, según CNAE 2009, han sido los siguientes: Agricultura (grupo A), Industria (grupos B, C, D y E), Comercio (G) y Servicios (H, J y U). En esta población se ha favorecido deliberadamente la participación de empresas destacadas en cada sector según la Central de Balances de Andalucía, base de datos oficial de la región, con el fin de aumentar el valor del análisis exploratorio de la investigación, en la medida que presta una atención preferente al grupo de organizaciones más influyentes en el mercado y la competencia sectorial. Este tipo de metodologías es habitual en estudios empíricos de tipo exploratorio que contrastan hipótesis y modelos explicativos sobre empresas en contextos específicos (Montalvo, 2006; Martínez-Román et al., 2011).

A partir de los datos disponibles en la Central de Balances de Andalucía y la base de empresas Bureau Van Dijk se elaboró una base de datos de 20.865 empresas andaluzas con una plantilla superior a 17 empleados. La recopilación de datos se realizó mediante encuestas telefónicas con cuestionario asistidas por ordenador (CATI) dirigidas a empresarios y CEOs de las empresas seleccionadas, con una tasa de respuesta del $11 \%$. Tras depurar los datos del trabajo de campo se obtuvo la base de datos utilizada en el análisis empírico. Eliminando empresas con un número de datos faltantes o anómalos se obtuvo la muestra final cuyo tamaño y estructura aparecen representados por sectores y provincias en la Tabla 1. 
Tabla 1

Estructura de la muestra

\begin{tabular}{|c|c|c|c|c|c|c|c|c|c|c|}
\hline Sectores & $\begin{array}{c}\text { CNAE } \\
\mathbf{2 0 0 9}\end{array}$ & Almería & Cádiz & Córdoba & Granada & Huelva & Jaen & Málaga & Sevilla & $\begin{array}{c}\text { Total } \\
\text { sector }\end{array}$ \\
\hline Agrario & $\mathrm{A}$ & 12 & 12 & 13 & 10 & 14 & 14 & 11 & 14 & 100 \\
\hline Industria & $\mathrm{B}, \mathrm{C}, \mathrm{D}, \mathrm{E}$ & 14 & 15 & 12 & 16 & 14 & 14 & 14 & 15 & 114 \\
\hline Comercio & $\mathrm{G}, \mathrm{H}^{\star}$ & 13 & 13 & 14 & 14 & 14 & 12 & 13 & 13 & 106 \\
\hline Servicios & $\mathrm{H}^{\star \star}, \mathrm{J}-\mathrm{U}$ & 13 & 14 & 14 & 14 & 14 & 14 & 13 & 14 & 110 \\
\hline $\begin{array}{c}\text { Total } \\
\text { provincia }\end{array}$ & & 52 & 54 & 53 & 54 & 56 & 54 & 51 & 56 & 430 \\
\hline
\end{tabular}

* Sólo los subgrupos de H: 4920, 4941, 5020, 5040, 5121, 5210, y 5224.

** Resto de subgrupos de $\mathrm{H}$.

Fuente: Elaboración propia.

\subsection{Variables}

En la Tabla 2 aparecen agrupadas por categorías las 35 variables explicativas seleccionadas para el contraste empírico del modelo y las variables a explicar, referidas a los resultados innovadores de las empresas. La exploración previa de asimetría y curtosis mostró una forma razonable en todas las variables, salvo en edad y tamaño, cuyo comportamiento log-normal aconsejó la transformación logarítmica en ambos casos. Tampoco se detectaron problemas relevantes de colinealidad entre las variables explicativas seleccionadas para el contraste del modelo.

Tabla 2

Variables del modelo

\begin{tabular}{|c|c|c|c|}
\hline \multicolumn{4}{|c|}{ VARIABLES EXPLICATIVAS } \\
\hline Categorías & & Descripción & Escalas \\
\hline \multirow{18}{*}{ 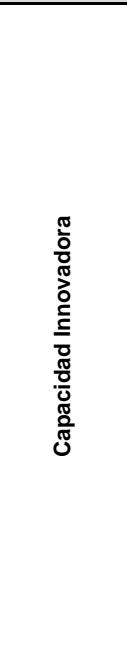 } & Conocimiento & & \\
\hline & Aprendizaje y capacitación & Importancia del aprendizaje en el puesto de trabajo & ordinal $(0-6)$ \\
\hline & & Asistencia a ferias, congresos y cursos especializados & ordinal $(0-6)$ \\
\hline & & Asistencia a ferias, congresos y cursos resto del personal & ordinal (0-6) \\
\hline & $\begin{array}{l}\text { Investigación y } \\
\text { experimentación }\end{array}$ & Patentes y otra propiedad industrial e intelectual & dicotómica \\
\hline & & Porcentaje del presupuesto anual en I+D y experimentación & numérica \\
\hline & Recursos humanos & Porcentaje de universitarios entre los directivos & numérica \\
\hline & & Porcentaje de universitarios entre no directivos & numérica \\
\hline & & La creatividad como criterio de promoción y recompensa & ordinal $(0-6)$ \\
\hline & & Nivel de riesgo asumido en nuevos proyectos & ordinal (0-6) \\
\hline & Organización & & \\
\hline & Nivel de autonomía & Nivel de autonomía en la toma de decisiones de los directivos & ordinal $(0-6)$ \\
\hline & & Nivel de autonomía en la toma de decisiones de no directivos & ordinal (0-6) \\
\hline & Comunicación & Existencia de grupos de trabajo permanentes & dicotómica \\
\hline & & Existencia de equipos especializados en cuestiones críticas & dicotómica \\
\hline & Jerarquía & Nivel de supervisión y control & ordinal $(0-6)$ \\
\hline & Estrategia & Estrategia competitiva (en la escala coste-diferenciación) & ordinal (0-6) \\
\hline & Calidad & Sistema de gestión de la calidad certificado & dicotómica \\
\hline
\end{tabular}


Tabla 2 (Continuación)

Variables del modelo

\begin{tabular}{|c|c|c|c|}
\hline \multicolumn{4}{|c|}{ VARIABLES EXPLICATIVAS } \\
\hline Categorías & & Descripción & Escalas \\
\hline \multirow{12}{*}{ 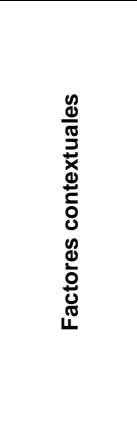 } & Tamaño & Número de trabajadores en plantilla & numérica \\
\hline & Financiación & Importancia de la autofinanciación & ordinal (0-6) \\
\hline & & Importancia de la financiación bancaria a corto plazo (<1 año) & ordinal $(0-6)$ \\
\hline & & Importancia de la financiación bancaria a medio plazo (1 a 5 años) & ordinal (0-6) \\
\hline & & Importancia de la financiación bancaria a largo plazo (>5 años) & ordinal $(0-6)$ \\
\hline & Cooperación & Con proveedores & ordinal $(0-6)$ \\
\hline & & Con distribuidores & ordinal (0-6) \\
\hline & & Con clientes & ordinal (0-6) \\
\hline & & Con redes de empresas & ordinal $(0-6)$ \\
\hline & & Con los competidores & ordinal $(0-6)$ \\
\hline & & Con universidades, laboratorios o centros tecnológicos & ordinal $(0-6)$ \\
\hline & & Con otras empresas & ordinal $(0-6)$ \\
\hline \multirow{7}{*}{ 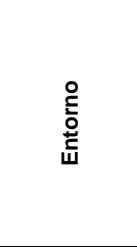 } & Competencia & Nivel de rivalidad competitiva en el mercado (hostilidad) & ordinal (0-6) \\
\hline & & Frecuencia de los cambios en la competencia (dinamismo) & ordinal $(0-6)$ \\
\hline & Soporte institucional & Importancia de subvenciones y ayudas a la innovación & ordinal (0-6) \\
\hline & & Opinión general sobre el régimen fiscal de la innovación & ordinal $(0-6)$ \\
\hline & & $\mathrm{N}^{\circ}$ de deducciones practicadas en el IS por I+D desde el 2000 & numérica \\
\hline & & Importancia de las deducciones en IS por I+D para la empresa & ordinal (0-6) \\
\hline & & Importancia de políticas y organismos de apoyo a exportación & ordinal $(0-6)$ \\
\hline \multicolumn{4}{|c|}{ VARIABLES A EXPLICAR } \\
\hline Categorías & & Descripción & Escalas \\
\hline \multirow{2}{*}{ 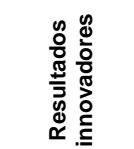 } & Innovación en productos & Nivel de novedad de innovaciones en productos realizadas & ordinal (0-4) \\
\hline & Innovación en procesos & Nivel de novedad de innovaciones en procesos realizadas & ordinal (0-4) \\
\hline
\end{tabular}

Fuente: Elaboración propia.

\section{RESULTADOS}

\subsection{Modelos sectoriales}

Para el contraste del modelo en cada sector se ha utilizado un modelo de regresión lineal múltiple del siguiente tipo:

$$
Y=c_{1} g_{1}\left(x_{1}\right)+c_{2} g_{2}\left(x_{2}\right)+\ldots+c_{n} g_{n}\left(x_{n}\right)+C_{0}
$$

El procedimiento se realizó aplicando previamente el método Backwards en cada una de las 8 regresiones lineales formuladas, es decir, una para productos y otra para procesos en cada uno de los 4 sectores investigados (Agrario, Industria, Comercio y Servicios). A partir del modelo inicial de 35 variables, este procedimiento estándar facilita la determinación del número óptimo de variables explicativas por sector y tipo de innovación, obteniendo el resultado final mostrado en las Tablas 3 y 4. En el sector Agrario, el modelo tiene 8 
variables para explicar la innovación en productos, siendo 5 significativas, y 17 para la innovación en procesos, de las que 8 son significativas; en el sector industrial, el modelo está formado por 13 variables explicativas para la innovación en productos y por 17 variables explicativas para la innovación en procesos, siendo 7 y 10 las variables significativas, respectivamente; en el Comercio, 12 y 13, siendo 7 las variables significativas en ambos casos; y en Servicios, los modelos contienen 15 y 20 variables explicativas, respectivamente, siendo 11 significativas en productos y 12 en el caso de los procesos.

\section{Tabla 3}

Coeficientes de regresión de los modelos sectoriales de innovación en productos

\begin{tabular}{|c|c|c|c|c|c|c|c|c|c|c|c|c|}
\hline & \multicolumn{3}{|c|}{ Agricultura } & \multicolumn{3}{|c|}{ Industria } & \multicolumn{2}{|c|}{ Comercio } & \multicolumn{4}{|c|}{ Servicios } \\
\hline & $b_{i}^{*}$ & $\mathbf{p}_{\mathrm{i}}$ & & $b_{i}^{*}$ & $\mathbf{p}_{\mathrm{i}}$ & & $b_{i}^{*}$ & $\mathbf{p}_{\mathrm{i}}$ & & $b_{i}^{*}$ & $\mathbf{p}_{\mathrm{i}}$ & \\
\hline Tamaño (número de empleados) & 0,113 & 0,279 & & & & & & & & 0,267 & 0,004 & $\left({ }^{\star *}\right)$ \\
\hline Autofinanciación & $-0,135$ & 0,197 & & & & & $-0,211$ & 0,018 & $(\star \star)$ & & & \\
\hline Ptmos. a C/P & $-0,199$ & 0,062 & $\left({ }^{\star}\right)$ & $-0,210$ & 0,139 & & & & & 0,313 & 0,004 & $(* \star)$ \\
\hline Ptmos. a M/P & & & & 0,443 & 0,009 & $(* *)$ & & & & $-0,372$ & 0,001 & $(* \star)$ \\
\hline Ptmos. A L/P & & & & $-0,158$ & 0,192 & & & & & & & \\
\hline \multicolumn{13}{|l|}{ Colab. proveedores } \\
\hline \multicolumn{13}{|l|}{ Colab. distribuidores } \\
\hline Colaboración clientes & & & & & & & & & & 0,139 & 0,128 & \\
\hline \multicolumn{13}{|l|}{ Colab. redes empresa } \\
\hline \multicolumn{13}{|l|}{ Colab. competidores } \\
\hline Coop. Universidades & $-0,255$ & 0,052 & $\left({ }^{\star}\right)$ & & & & & & & 0,210 & 0,062 & $\left({ }^{\star}\right)$ \\
\hline \multicolumn{13}{|l|}{ Coop. otras empresas } \\
\hline Certiflcación de calidad & & & & & & & & & & $-0,149$ & 0,094 & $\left({ }^{\star}\right)$ \\
\hline Aprendizaje trabajo & & & & & & & 0,180 & 0,056 & $\left({ }^{\star}\right)$ & & & \\
\hline \multicolumn{13}{|l|}{ Asist. ferias/congresos directivos } \\
\hline Asist. ferias/congres. no directivos & & & & $-0,201$ & 0,043 & $(\star \star)$ & & & & $-0,217$ & 0,027 & $\left({ }^{\star \star}\right)$ \\
\hline Patentes y otra prop. Intelectual & 0,308 & 0,013 & $(* \star)$ & 0,190 & 0,041 & $(* *)$ & & & & & & \\
\hline Esfuerzo interno en I+D & & & & 0,348 & 0,001 & $(* \star)$ & 0,358 & 0,000 & $(* \star)$ & & & \\
\hline$\%$ Universitarios directivos & & & & $-0,147$ & 0,130 & & & & & 0,212 & 0,022 & $(* \star)$ \\
\hline$\%$ Universitario no directivos & & & & & & & 0,218 & 0,015 & $(\star \star)$ & $-0,156$ & 0,124 & \\
\hline Creatividad & & & & 0,157 & 0,113 & & 0,103 & 0,282 & & 0,202 & 0,039 & $\left({ }^{\star \star}\right)$ \\
\hline Tolerancia al riesgo & & & & 0,103 & 0,281 & & & & & & & \\
\hline Autonomía directivos & & & & & & & $-0,187$ & 0,044 & $(\star \star)$ & 0,155 & 0,097 & $\left({ }^{\star}\right)$ \\
\hline Autonomía no directivos & & & & & & & 0,149 & 0,118 & & 0,230 & 0,034 & $\left({ }^{\star \star}\right)$ \\
\hline Grupos de trabajo & & & & & & & $-0,167$ & 0,060 & $\left({ }^{\star}\right)$ & 0,108 & 0,264 & \\
\hline Equipos especializados & & & & 0,105 & 0,247 & & & & & & & \\
\hline Supervisión y control & & & & & & & & & & 0,150 & 0,088 & $\left({ }^{\star}\right)$ \\
\hline \multicolumn{13}{|l|}{ Orientación estratégica } \\
\hline Rivalidad competitiva & & & & $-0,241$ & 0,025 & $(* \star)$ & & & & & & \\
\hline Dinamismo mercado & & & & 0,199 & 0,059 & $\left({ }^{*}\right)$ & 0,110 & 0,247 & & & & \\
\hline Subvenciones públicas & 0,145 & 0,211 & & & & & & & & 0,138 & 0,179 & \\
\hline Régimen fiscal I+D & $-0,193$ & 0,052 & $(*)$ & & & & $-0,138$ & 0,117 & & & & \\
\hline No deducciones I+D en IS & 0,288 & 0,026 & $(\star \star)$ & & & & $-0,126$ & 0,159 & & & & \\
\hline \multicolumn{13}{|l|}{ Importe deducciones I+D en IS } \\
\hline Ayudas exportación & & & & 0,189 & 0,049 & $(\star \star)$ & 0,175 & 0,054 & $\left({ }^{\star}\right)$ & & & \\
\hline Coeficiente $\mathrm{R}^{\prime 2}$ & 0,221 & & & 0,344 & & & 0,346 & & & 0,351 & & \\
\hline p-value regresión & $<0.1 \%$ & & & $<0.1 \%$ & & & $<0.1 \%$ & & & $<0.1 \%$ & & \\
\hline$* 0.05<p$-value $\leq 0,1$ & & & & & & & & & & & & \\
\hline$\star *$-value $\leq 0,05$ & & & & & & & & & & & & \\
\hline
\end{tabular}

Fuente: Elaboración propia. 
Tabla 4

Coeficientes de regresión de los modelos sectoriales de innovación en procesos

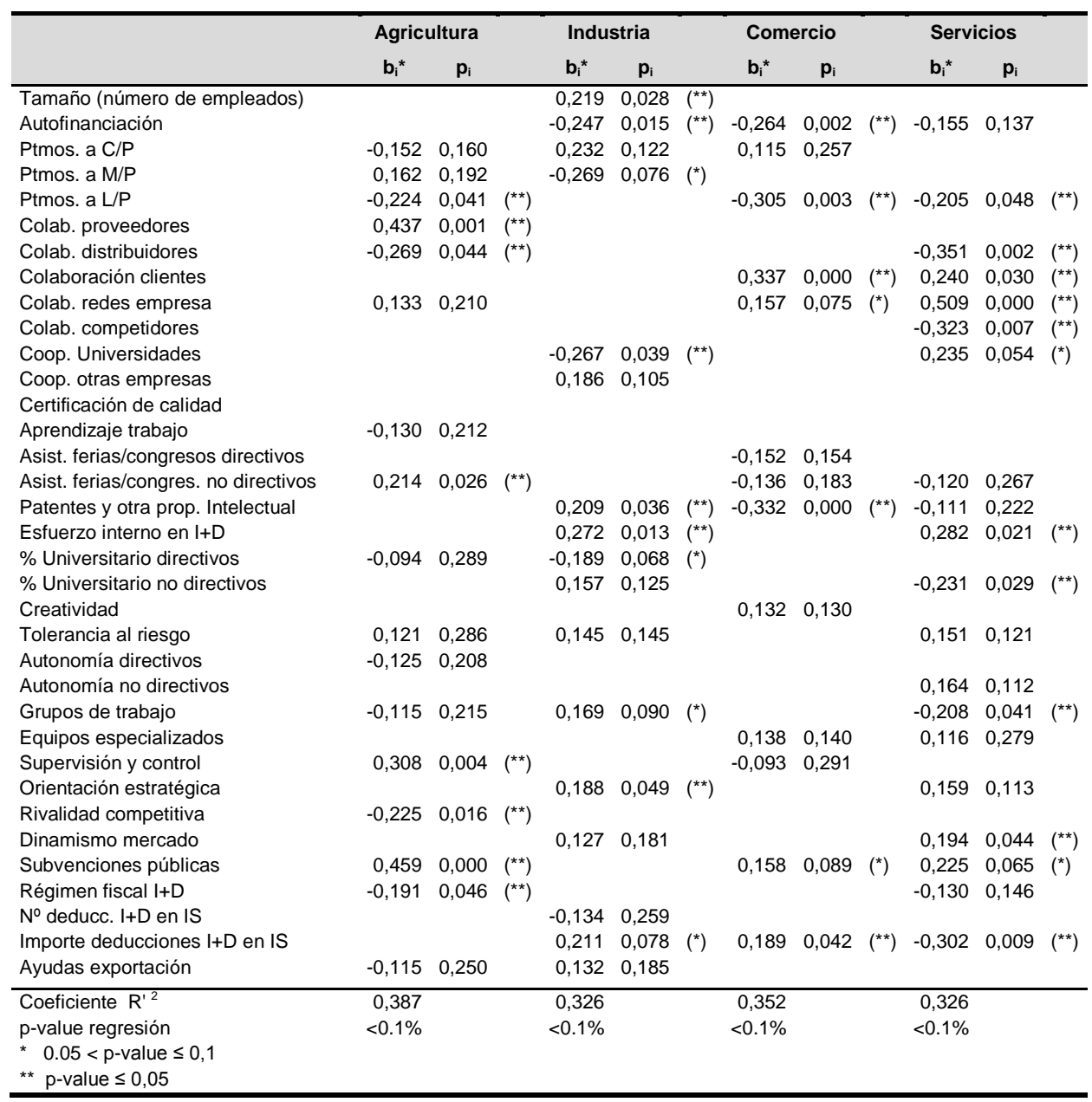

Fuente: Elaboración propia.

Para el contraste empírico se ha aplicado el test de significación F-Snedecor a cada regresión múltiple de variables explicativas sobre los resultados de innovación. Los resultados del test y los p-value de los modelos en cada sector para la innovación en productos aparecen en la Tabla 3 y para la innovación en procesos en la Tabla 4. Como puede observarse en ambas tablas, todas las regresiones superan el test de significación $(\mathrm{p}<0.1 \%)$, quedando así contrastadas las hipótesis $\mathrm{H} 1$ y $\mathrm{H} 2$ en todos los sectores y tipo de innovación. Asimismo, la capacidad explicativa de los modelos de innovación en productos ronda el 35\%, salvo en el sector agrario que se queda en el $22 \%$, mientras que para la 
innovación en procesos se halla entre el 32\% y el 38\%, según los casos. Por tanto, podemos afirmar que los modelos poseen una capacidad explicativa ajustada a las características del estudio, aunque con diferencias sectoriales. Estas diferencias morfológicas entre los modelos sectoriales, reflejadas en sus conjuntos de variables explicativas, ponen de manifiesto las peculiaridades de la innovación en cada grupo de actividades productivas.

\subsection{Tipología empresa innovadora vs. no innovadora}

\subsubsection{Análisis descriptivo}

A partir de la información proporcionada por estos modelos sectoriales se ha podido caracterizar a la empresa innovadora respecto a la empresa que no innova en la región de Andalucía. La Tabla 5 recoge un estudio previo de la distribución de frecuencias en ambos casos por tipo de innovación de forma general y por sectores. En el caso de las novedades en productos, el 65,2\% de las empresas declararon no haber realizado ninguna innovación de este tipo en los últimos tres años, siendo el agrario el sector menos innovador, mientras que la menor proporción de organizaciones no innovadoras corresponde a la industria. Por el contrario, sólo el 37,4\% del total de empresas encuestadas no innovaron en procesos, siendo de nuevo el sector agrario la actividad con mayor proporción de empresas no innovadoras, situándose de nuevo la actividad industrial en el extremo opuesto.

Tabla 5

Número y frecuencia de empresas innovadoras y no innovadoras en la muestra

\begin{tabular}{|c|c|c|c|c|c|c|c|c|c|c|c|}
\hline \multirow[t]{2}{*}{ Tipo } & \multirow[t]{2}{*}{ Nivel } & \multicolumn{2}{|c|}{ Total } & \multicolumn{2}{|c|}{ Agrario } & \multicolumn{2}{|c|}{ Industria } & \multicolumn{2}{|c|}{ Comercio } & \multicolumn{2}{|c|}{ Servicios } \\
\hline & & $\mathbf{N}^{\circ}$ & Frec. & $\mathbf{N}^{\circ}$ & Frec. & $\mathbf{N}^{\circ}$ & Frec. & $\mathrm{N}^{\circ}$ & Frec. & $\mathbf{N}^{\circ}$ & Frec. \\
\hline \multirow{6}{*}{ 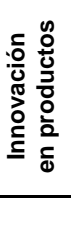 } & 0 & 271 & $41,6 \%$ & 76 & $76,0 \%$ & 59 & $51,8 \%$ & 72 & $67,9 \%$ & 64 & $58,2 \%$ \\
\hline & 1 & 39 & $6,0 \%$ & 6 & $6,0 \%$ & 7 & $6,1 \%$ & 9 & $8,5 \%$ & 17 & $15,5 \%$ \\
\hline & 2 & 40 & $6,1 \%$ & 6 & $6,0 \%$ & 17 & $14,9 \%$ & 10 & $9,4 \%$ & 7 & $6,4 \%$ \\
\hline & 3 & 50 & $7,7 \%$ & 7 & $7,0 \%$ & 22 & $19,3 \%$ & 10 & $9,4 \%$ & 11 & $10,0 \%$ \\
\hline & 4 & 30 & $4,6 \%$ & 5 & $5,0 \%$ & 9 & $7,9 \%$ & 5 & $4,7 \%$ & 11 & $10,0 \%$ \\
\hline & Totales & 430 & $66 \%$ & 100 & $100 \%$ & 114 & $100 \%$ & 106 & $100 \%$ & 110 & $100 \%$ \\
\hline \multirow{6}{*}{ 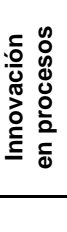 } & 0 & 145 & $22,2 \%$ & 41 & $41,0 \%$ & 33 & $28,9 \%$ & 36 & $34,0 \%$ & 35 & $31,8 \%$ \\
\hline & 1 & 24 & $3,7 \%$ & 7 & $7,0 \%$ & 7 & $6,1 \%$ & 5 & $4,7 \%$ & 5 & $4,5 \%$ \\
\hline & 2 & 173 & $26,5 \%$ & 47 & $47,0 \%$ & 61 & $53,5 \%$ & 35 & $33,0 \%$ & 30 & $27,3 \%$ \\
\hline & 3 & 76 & $11,7 \%$ & 4 & $4,0 \%$ & 8 & $7,0 \%$ & 29 & $27,4 \%$ & 35 & $31,8 \%$ \\
\hline & 4 & 12 & $1,8 \%$ & 1 & $1,0 \%$ & 5 & $4,4 \%$ & 1 & $0,9 \%$ & 5 & $4,5 \%$ \\
\hline & Totales & 430 & $66 \%$ & 100 & $100 \%$ & 114 & $100 \%$ & 106 & $100 \%$ & 110 & $100 \%$ \\
\hline
\end{tabular}

Fuente: Elaboración propia.

La representación gráfica de la Figura 2 muestra una clara diferenciación entre las empresas innovadoras y no innovadoras en productos, mientras que 
esta dicotomía no resulta tan evidente en la innovación en procesos, debido a la presencia de novedades tipo 2 y 3 en el conjunto de empresas. En el eje horizontal se representan los valores de la escala de innovación en productos y procesos (0-4) y en el eje vertical el número de empresas para cada nivel de la escala.

Figura 2

Perfil de empresas innovadoras y no innovadoras

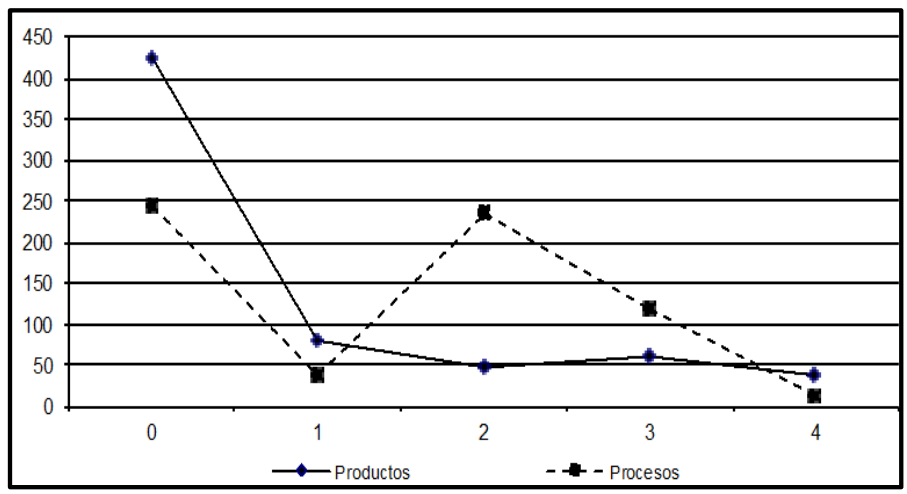

Fuente: Elaboración propia.

También se aprecian diferencias sectoriales en la distribución de las empresas innovadoras, tanto en productos como en procesos. Estas diferencias quedan patentes en los perfiles de innovación representados en la Figura 3, para la innovación en productos, y en la Figura 4, en el caso de la innovación en procesos. Como puede observarse, la mayor radicalidad en productos corresponde a la industria y los servicios (niveles 4 y 3 de la escala de novedades), en el caso de modificaciones leves la industria es el sector principal sector (nivel 2) y cuando el grado de novedad es mínimo destaca el sector servicios (nivel 1). Cuando se trata de procesos totalmente nuevos en el mercado predominan por igual la industria y los servicios (nivel 4 en la escala de innovaciones en procesos). Por otro lado, la mayor parte de las novedades en sistemas de información y comunicación se producen en los servicios y el comercio (nivel 3), mientras que el predominio de la industria y el sector agrario es claro en la adquisición de nueva tecnología incorporada en maquinaria especializada (nivel 2). Por último, las pequeñas innovaciones en procesos fruto de la experiencia surgen por igual en todos los sectores productivos. 
Figura 3

Perfil de empresas innovadoras en productos

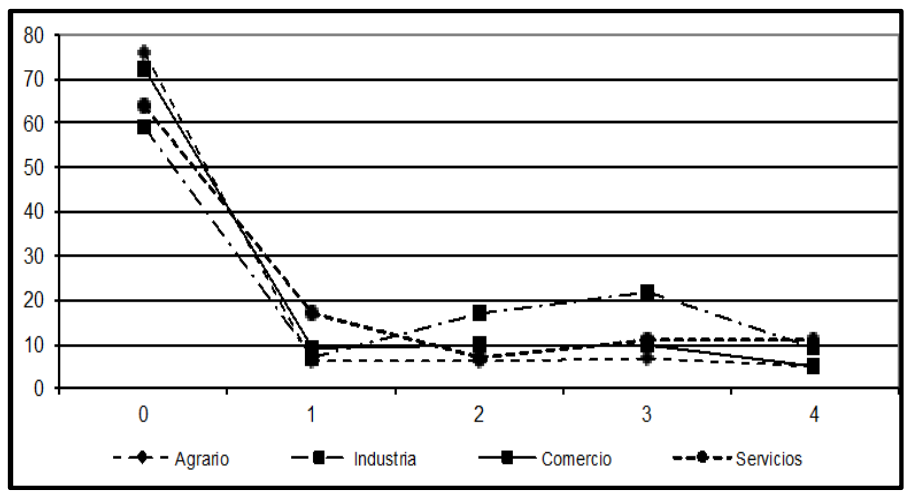

Fuente: Elaboración propia.

Figura 4

Perfil de empresas innovadoras en procesos

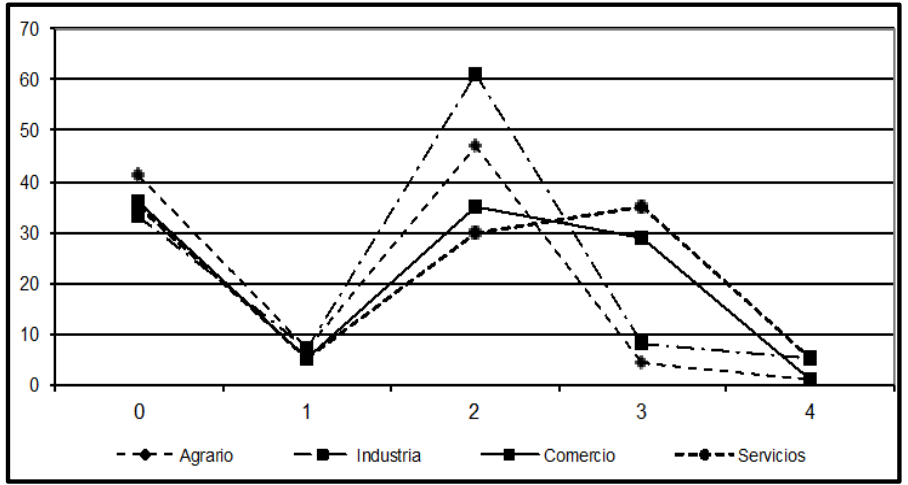

Fuente: Elaboración propia.

\subsubsection{Elaboración de la tipología}

El procedimiento seguido para la clasificación de empresas comienza formando dos grupos con todos los elementos muestrales: empresas innovadoras y no innovadoras, y seleccionando del conjunto inicial de variables explicativas aquellas cuyos coeficientes en los modelos anteriores han sido significativos al menos en dos sectores $(\mathrm{p}<0,05)$. Dicha selección ha sido completada con variables del modelo de especial relevancia en otros sectores estratégicos de esta región, como son el turismo (Martínez-Román et al., 2015) y la construcción (Martínez-Román et al., 2017), con el fin de ofrecer una panorámica lo más 
amplia posible de las empresas innovadoras andaluzas ${ }^{1}$. El conjunto final de variables para la elaboración de la tipología general de empresa innovadora aparece en la Tabla 6, para los productos, y en la Tabla 7, para los procesos.

Tabla 6

Intervalo de Tchebychev variables significativas para innovación en productos

\begin{tabular}{|c|c|c|c|c|c|c|c|c|}
\hline \multirow{3}{*}{$\begin{array}{l}\text { Variables } \\
\text { cia ferias y cong. no } \\
\text { dir. }\end{array}$} & \multirow{3}{*}{$\begin{array}{c}\text { Escala } \\
(0-6)\end{array}$} & \multicolumn{2}{|c|}{ Innovadoras } & \multicolumn{2}{|c|}{ No innovadoras } & \multirow{2}{*}{\multicolumn{2}{|c|}{$\begin{array}{c}\text { Intervalo } \\
m_{2} \pm 2 s_{2}\end{array}$}} & \multirow{3}{*}{$\begin{array}{c}\text { Significat. } \\
\text { Sí }\end{array}$} \\
\hline & & \multirow{2}{*}{$\begin{array}{c}\begin{array}{c}\text { Media } \\
\left(\mathbf{m}_{1}\right)\end{array} \\
2,7723\end{array}$} & \multirow{2}{*}{$\begin{array}{c}\begin{array}{c}\text { Desviac. } \\
\left(\mathbf{s}_{1}\right)\end{array} \\
0,1331\end{array}$} & \multirow{2}{*}{$\begin{array}{c}\begin{array}{c}\text { Media } \\
\left(\mathbf{m}_{2}\right)\end{array} \\
2,4048\end{array}$} & \multirow{2}{*}{$\begin{array}{c}\begin{array}{c}\text { Desviac. } \\
\left(\mathbf{s}_{2}\right)\end{array} \\
0,1047\end{array}$} & & & \\
\hline & & & & & & 2,1954 & 2,6142 & \\
\hline $\begin{array}{l}\text { Patentes y otra Prop. } \\
\text { Industrial }\end{array}$ & $(0-1)$ & 0,2423 & 0,0285 & 0,0753 & 0,0128 & 0,0497 & 0,1009 & Sí \\
\hline Tamaño & Numérica & 3,8787 & 0,0619 & 3,5977 & 0,0362 & 3,5252 & 3,6701 & Sí \\
\hline Creatividad & $(0-6)$ & 3,3502 & 0,1335 & 2,3624 & 0,1089 & 2,1445 & 2,5802 & Sí \\
\hline Préstamos a corto plazo & $(0-6)$ & 3,2699 & 0,1356 & 3,1098 & 0,1047 & 2,9004 & 3,3191 & No \\
\hline Préstamos a medio plazo & $(0-6)$ & 2,8319 & 0,1312 & 2,8993 & 0,1020 & 2,6952 & 3,1033 & No \\
\hline Esfuerzo en I+D & Numérica & 1,3062 & 0,1016 & 0,4556 & 0,0487 & 0,3583 & 0,5529 & Sí \\
\hline$\%$ Universitarios directivos & Numérica & 60,6102 & 2,5730 & 54,9151 & 1,9322 & 51,0507 & 58,7795 & Sí \\
\hline Autonomía directivos & $(0-6)$ & 4,9075 & 0,0806 & 5,0259 & 0,0576 & 4,9108 & 5,1411 & No \\
\hline $\mathrm{N}^{0}$ deducciones I+D en IS & Numérica & 1,2291 & 0,1742 & 0,4188 & 0,0715 & 0,2758 & 0,5618 & Sí \\
\hline Apoyo a la exportación & $(0-6)$ & 0,8973 & 0,1125 & 0,3656 & 0,0561 & 0,2534 & 0,4778 & Sí \\
\hline
\end{tabular}

Fuente: Elaboración propia.

A continuación, se midió su valor discriminante o clasificador, calculando los centroides de cada variable seleccionada (media: m) en ambos grupos de empresas, innovadoras y no innovadoras, y sus respectivos errores muestrales según el tamaño del grupo (desviación típica: s). Estos datos han permitido formar unos clústeres mediante la aplicación del intervalo de Tchebychev $(\mathrm{m} \pm$ 2s), comprobándose si los clústeres se solapan o no en cada caso. Cuando dicho solapamiento no se produce, o es pequeño, se considera que la variable permite clasificar entre innovadoras y no innovadoras, en caso contrario la variable no es significativa para discriminar entre ambos grupos de empresas. Las Tablas 6 y 7 contienen los resultados obtenidos para cada variable, tanto en productos como en procesos, teniendo en cuenta que las medias y desviaciones típicas calculadas deben ser interpretadas en función del rango de valores de su escala de medida. Una variable será significativa, es decir, poseerá capacidad discriminante, cuando el centroide del grupo innovador no pertenezca al intervalo de valores del grupo no innovador ( $\mathrm{m} 2 \pm 2 \mathrm{~s} 2$ ), o viceversa.

\footnotetext{
${ }^{1}$ Este criterio adicional ha supuesto la incorporación del tamaño, el porcentaje de directivos universitarios, la creatividad y la deducción de gastos en I+D en el Impuesto de Sociedades, en el caso de la innovación en productos, y de la colaboración con competidores, las asistencias a ferias y congresos, la supervisión y control, la orientación estratégica y el dinamismo del mercado, para la innovación en procesos. La mayoría de estas variables también han sido significativas al menos en uno de los sectores productivos analizados en esta investigación.
} 
Tabla 7

Intervalo de Tchebychev variables significativas para innovación en procesos

\begin{tabular}{|c|c|c|c|c|c|c|c|c|}
\hline \multirow[b]{2}{*}{ Variables } & \multirow[b]{2}{*}{ Escala } & \multicolumn{2}{|c|}{ Innovadoras } & \multicolumn{2}{|c|}{ No innovadoras } & \multirow{2}{*}{\multicolumn{2}{|c|}{$\begin{array}{c}\text { Intervalo } \\
m_{2} \pm 2 s_{2}\end{array}$}} & \multirow[b]{2}{*}{ Significat. } \\
\hline & & $\begin{array}{l}\text { Media } \\
\left(\mathrm{m}_{1}\right)\end{array}$ & $\begin{array}{l}\text { Desviac. } \\
\left(\mathrm{s}_{1}\right)\end{array}$ & $\begin{array}{l}\text { Media } \\
\left(\mathrm{m}_{2}\right)\end{array}$ & $\begin{array}{l}\text { Desviac. } \\
\left(s_{2}\right)\end{array}$ & & & \\
\hline Autofinanciación & $(0-6)$ & 4,6373 & 0,0756 & 4,5943 & 0,1095 & 4,3753 & 4,8132 & No \\
\hline Esfuerzo en I+D & Numérica & 0,9811 & 0,0692 & 0,3682 & 0,0596 & 0,2490 & 0,4874 & Sí \\
\hline Préstamos a L/P & $(0-6)$ & 2,7037 & 0,1071 & 2,9056 & 0,1462 & 2,6131 & 3,1980 & No \\
\hline $\begin{array}{l}\text { Patentes y otra Prop. } \\
\text { Industrial }\end{array}$ & $(0-1)$ & 0,1422 & 0,0173 & 0,1189 & 0,0208 & 0,0773 & 0,1604 & No \\
\hline $\begin{array}{l}\text { Cooperación con } \\
\text { distribuidores }\end{array}$ & $(0-6)$ & 3,3701 & 0,1161 & 3,0082 & 0,1541 & 2,7000 & 3,3164 & Sí \\
\hline Cooperación con clientes & $(0-6)$ & 4,6373 & 0,0888 & 3,8683 & 0,1456 & 3,5772 & 4,1594 & Sí \\
\hline $\begin{array}{l}\text { Cooperación con } \\
\text { competidores }\end{array}$ & $(0-6)$ & 2,1946 & 0,0953 & 1,8025 & 0,1267 & 1,5490 & 2,0559 & Sí \\
\hline $\begin{array}{l}\text { Asistencia ferias y } \\
\text { congresos dir. }\end{array}$ & $(0-6)$ & 3,6757 & 0,0954 & 3,3580 & 0,1282 & 3,1017 & 3,6143 & Sí \\
\hline $\begin{array}{l}\text { Asistencia ferias y cong. no } \\
\text { dir. }\end{array}$ & $(0-6)$ & 2,5885 & 0,1045 & 2,4403 & 0,1354 & 2,1696 & 2,7111 & No \\
\hline Dinamismo mercado & $(0-6)$ & 3,9229 & 0,0869 & 3,9706 & 0,1141 & 3,7423 & 4,1989 & No \\
\hline $\begin{array}{l}\text { Importancia deducciones } \\
\text { por I+D }\end{array}$ & $(0-6)$ & 0,6786 & 0,0712 & 0,2438 & 0,0584 & 0,1271 & 0,3605 & Sí \\
\hline
\end{tabular}

Fuente: Elaboración propia.

Por ejemplo, la Asistencia a ferias y congresos de no directivos (ver Tabla 6) es una variable significativa o con capacidad discriminante porque su media (2.7723) queda fuera del intervalo de valores de esta variable en el conjunto de empresas no innovadoras de la muestra $(2.1954,2.6142)$. Del mismo modo, la Autofinanciación (ver Tabla 7) no es una variable significativa, es decir, no es válida para clasificar, ya que su media $(4,6373)$ pertenece al intervalo de valores de dicha variable en las empresas no innovadoras (4.3753, 4.8132). En el primer caso, el resultado permite afirmar que dicha fuente de conocimiento externo es más importante para las empresas innovadoras que para el resto, en la medida que dicho valor medio se halla próximo al valor 3 de la escala de medición de la variable, en el primer caso, y más cercano al valor 2 de la misma, en el segundo.

Las variables de la Tabla 6 aparecen representadas en la Figura 5 y las variables de la Tabla 7 en la Figura 6. Gráficamente se observa la ausencia de solapamiento de las variables significativas o, si aparecen, son mínimos, mientras que en caso contrario existe una gran intersección entre ambas circunferencias, incluso a veces una contiene a la otra. Además, cuanto mayor sea la distancia entre ambas circunferencias más clara es la diferencia en ese factor entre las empresas que innovan y las que no lo hacen.

La siguiente clasificación muestra las variables que han resultado discriminantes para la innovación en productos en cada una de las categorías del modelo:

- Capacidad innovadora. En la dimensión conocimiento han resultado significativas Asistencia a ferias y congresos de no directivos, Esfuerzo en I+D y Patentes y otra propiedad industrial e intelectual. En la dimensión 
de recursos humanos: Creatividad y porcentaje de universitarios entre los directivos. Por último, ninguna variable de la dimensión de organización ha sido significativa.

- Variables contextuales. Sólo la variable Tamaño es significativa.

- Variables del entorno. Han resultado ser significativas el Número de deducciones por I+D en el impuesto de sociedades y la Importancia de políticas y organismos de apoyo a la exportación, ambas con mayor valor en las empresas que innovan.

Figura 5

Centroides e intervalos de las variables para la innovación en productos
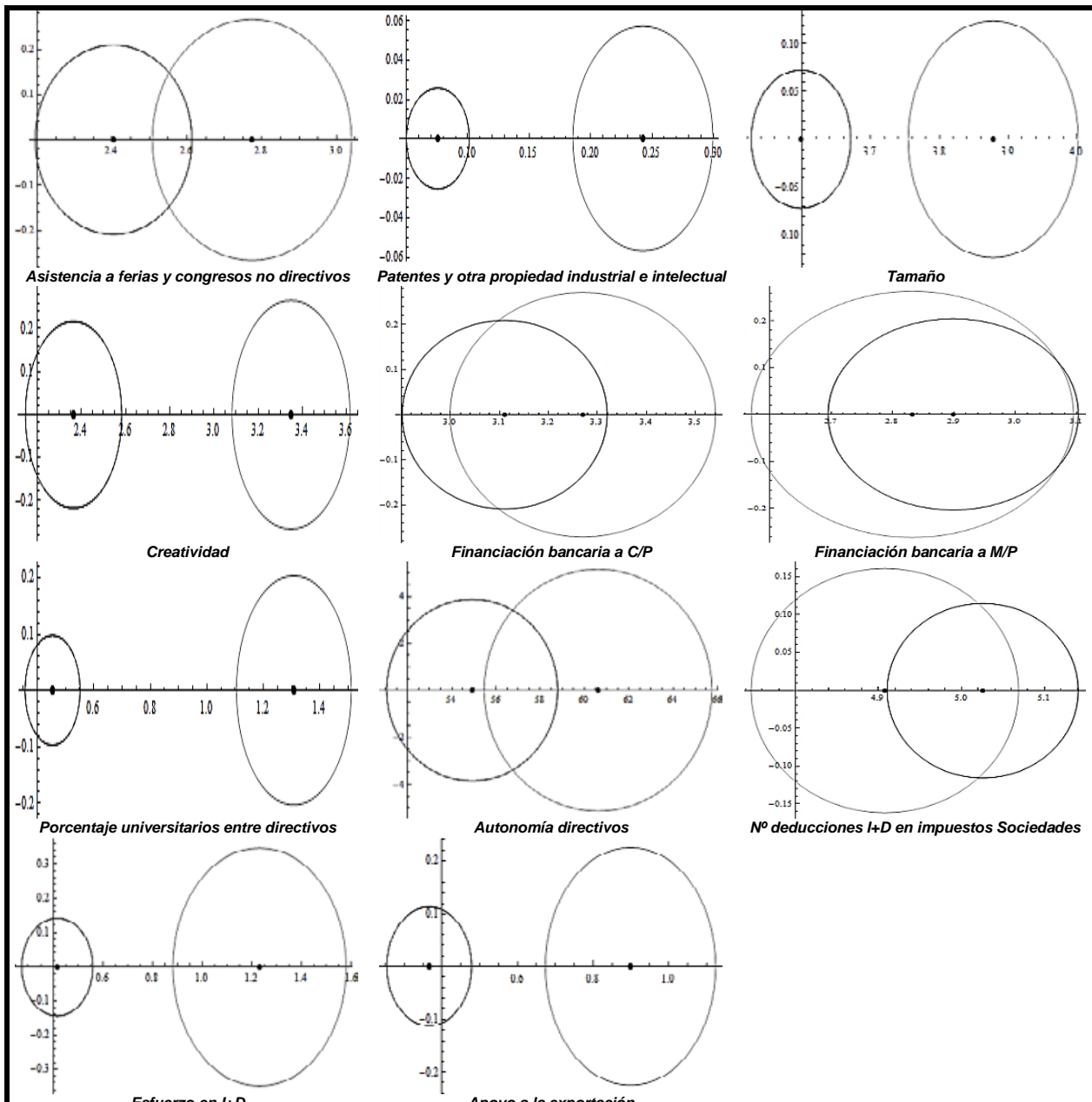

Patentes y otra propiedad industrial e intelectua
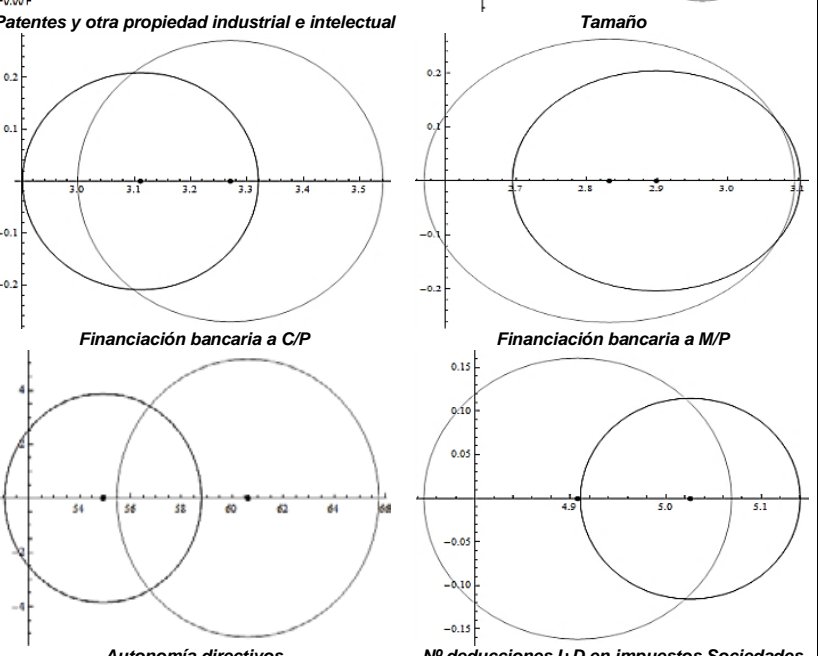

Esfuerzo en $I+D$

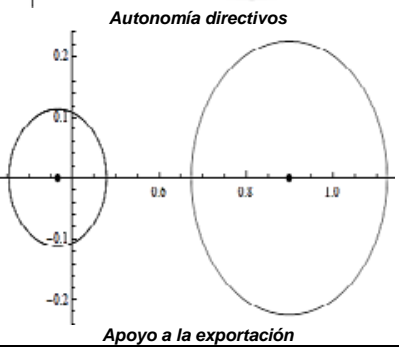

$N^{\circ}$ deducciones I+D en impuestos Sociedades

Fuente: Elaboración propia. 
Figura 6

Centroides e intervalos de las variables para la innovación en procesos

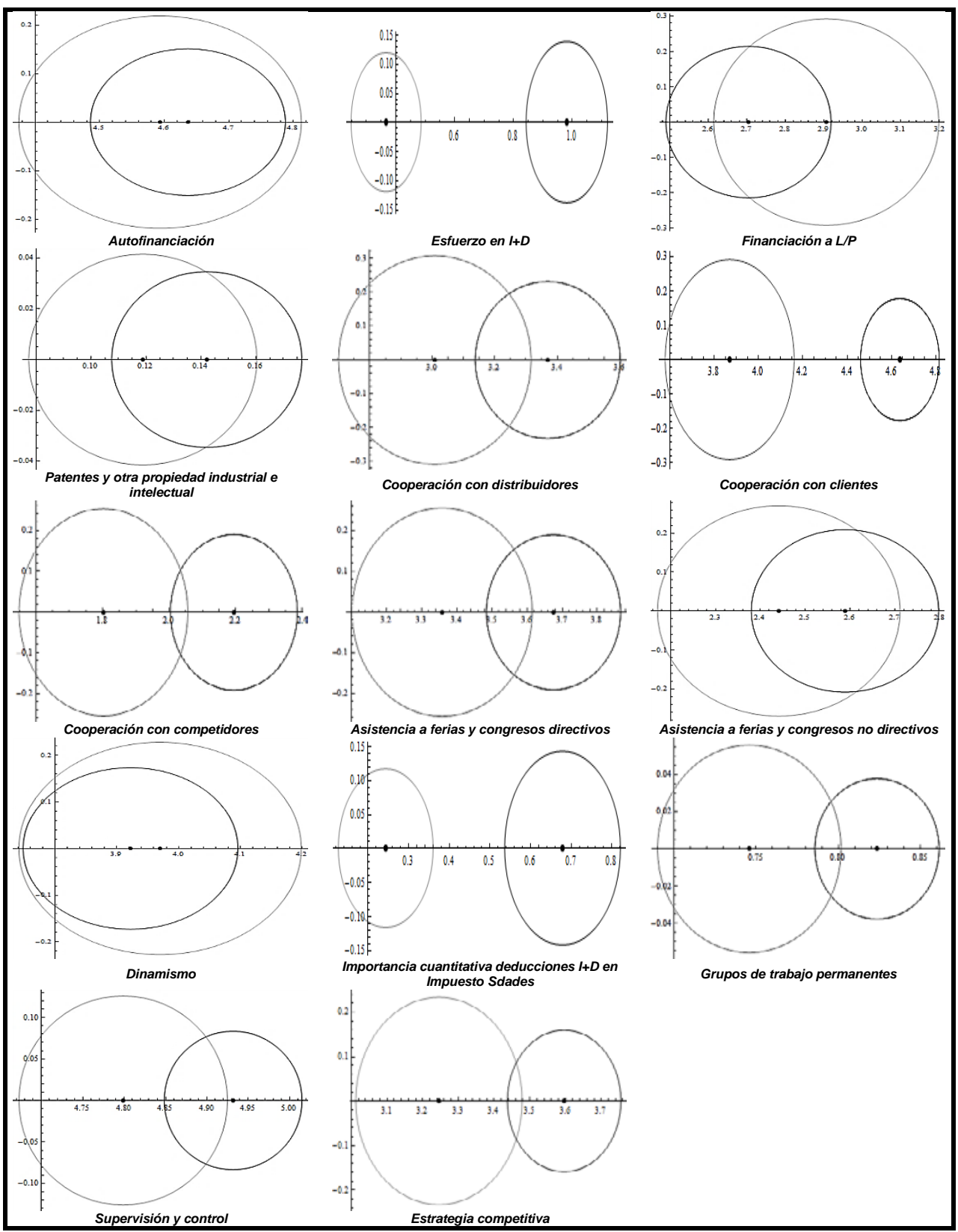

Fuente: Elaboración propia. 
Análogamente, los resultados para la innovación en procesos pueden resumirse del siguiente modo:

- Capacidad innovadora. En la dimensión conocimiento han demostrado ser significativas el Esfuerzo en I+D y la Asistencia a ferias y congresos de directivos; en la dimensión de organización los Grupos de trabajo permanentes, la Supervisión y control y la Estrategia competitiva; y ninguna en la dimensión de recursos humanos.

- Variables contextuales. En esta categoría han resultado significativas la Cooperación con distribuidores, clientes y competidores.

- Variables del entorno. Sólo ha resultado significativa la Importancia cuantitativa de las deducciones por I+D en el impuesto de sociedades.

Finalmente, la Tabla 8 (ver Apéndice) sintetiza toda la información anterior mostrando las características de la empresa innovadora frente a la no innovadora, situando en las columnas del extremo las variables significativas con más poder discriminante (sin solapamiento entre circunferencias) y en las más cercanas o próximas las variables significativas con menor poder discriminante (con cierto solapamiento entre circunferencias).

\section{DISCUSIÓN Y CONCLUSIONES}

Los resultados de este trabajo abogan por el desarrollo del análisis micronivel en el estudio de la innovación a nivel regional (Uyarra, 2010). En nuestro caso, este enfoque de investigación ha facilitado un modelo explicativo del nivel de innovación en los sectores más y menos tecnológicos de Andalucía, proporcionando así una tipología específica de la empresa innovadora en esta importante región periférica de la U.E.

El contraste de las hipótesis de investigación permite afirmar que los resultados innovadores en productos (H1) y en procesos (H2) pueden expresarse con un modelo lineal generalizado de variables explicativas referidas a la capacidad innovadora, los factores contextuales y el entorno. La validación de los 8 modelos lineales propuestos ha revelado unos patrones de innovación empresarial por cada sector (Agrario, Industria, Comercio y Servicios) y por tipo de innovación (productos y procesos) a nivel regional. Estos resultados corroboran la importancia que la literatura concede a la combinación de los enfoques regional y sectorial en el estudio espacial de la innovación (Breschi y Malerba, 1997).

El análisis de la tipología revela rasgos específicos asociados al tipo de innovación. La organización que innova en productos se caracteriza, independientemente del sector, por el desarrollo de una capacidad innovadora donde las fuentes de conocimiento interno (mayor esfuerzo en I+D y apropiación formal del conocimiento con patentes, etc.) predominan sobre las fuentes de 
carácter externo (asistencia a ferias y congresos), siendo fundamental también en este caso el factor humano (creatividad y porcentaje de universitarios directivos), la dimensión productiva de la empresa (tamaño) y las medidas de apoyo institucional a la innovación (deducciones fiscales a la I+D y el soporte de apoyo institucional a la exportación). De este modo, los resultados confirman la prevalencia de las fuentes internas en la innovación en productos (Koschatzky, 1998; Sternberg y Arndt, 2001), es decir, ponen de manifiesto la necesidad que tienen las empresas de desarrollar su propia capacidad innovadora para poder beneficiarse de la transferencia tecnológica desde su entorno (Kessler, Bierly y Gopalakrishnan, 2000; Oliveira y Martins, 2010). Tener un mayor tamaño proporciona las infraestructuras tecnológicas y los recursos productivos necesarios para poder desarrollar esta capacidad interna (Bhaskaran, 2006), especialmente en contextos con sistemas de innovación débiles donde la cooperación no es determinante para innovar (Martínez-Román et al., 2011), como es el caso. Por esta misma razón, la disponibilidad de recursos humanos en la organización favorece el nivel de innovación en productos en estas economías menos desarrolladas (Radas y Bozic, 2009). Por último, los resultados confirman el impulso que la ayuda pública directa, como son los beneficios fiscales a las actividades de I+D y el apoyo a la internacionalización, proporciona al fomento del comportamiento innovador de las empresas (Kaufmann y Tödtling, 2002; Kleer, 2010).

Por su parte, la organización que innova en procesos fundamenta su capacidad innovadora en la creación de conocimiento (esfuerzo en I+D) y en determinadas fuentes externas de conocimiento (asistencia a ferias y congresos de directivos), así como en elementos del diseño organizativo de la empresa (grupos de trabajo permanentes y, en menor grado, la supervisión y control y la estrategia de diferenciación. Es decir, los elementos estructurales adquieren gran importancia en este tipo de innovación (Menguc y Auh, 2010; Russell, 1990) frente a los atributos del personal, como puede ser su nivel de cualificación y su creatividad, que resultaban determinantes en el caso de la innovación en productos. Asimismo, el ambiente externo adquiere mayor importancia cuando se trata de la innovación en procesos. En efecto, la cooperación con clientes y, en menor grado, con distribuidores y competidores, proporcionan canales de información y cooperación de especial utilidad para implantar novedades en procesos en las empresas de la región. Este resultado coincide con el de otras investigaciones en las que la innovación en producto depende del conocimiento interno y la innovación de procesos tiene su principal origen en las fuentes externas de conocimiento (Hervás-Oliver et al., 2014; Martínez-Román et al., 2017). Asimismo, la principal influencia del entorno específico proviene nuevamente del soporte institucional público, concretamente de las deducciones fiscales a la I+D, insistiendo en la importancia de medidas de apoyo a la inversión en innovación frente a las subvenciones o la colaboración con universidades, KIBS 
y otros agentes, cuya importancia es grande en las regiones tecnológicamente avanzadas. Por tanto, los resultados destacan, junto a la creación de conocimiento interno, el papel relevante de los elementos organizativos (Hull y Covin, 2010), estratégicos (Martínez-Román et al., 2015) y del entorno (Hervás-Oliver et al., 2014) en la innovación en procesos de las empresas de la región.

Aunque el estudio y los resultados de esta investigación se limitan a la región de Andalucía, los instrumentos analíticos del trabajo pueden ser perfeccionados y utilizados en otros ámbitos regionales periféricos, facilitando así el análisis comparativo a nivel empresa y la extracción de conclusiones de carácter general sobre la innovación en este tipo de regiones.

\section{REFERENCIAS BIBLIOGRÁFICAS}

ASHEIM, B.T. y COENEN, L. (2005). "Knowledge Bases and Regional Innovation Systems: Comparing Nordic Clusters". Research Policy, 34(8), pp. 1173-1190.

BHASKARAN, S. (2006). "Incremental Innovation and Business Performance: Small and Medium-size Food Enterprises in a Concentrated Industry Environment". Journal of Small Business Management, 44(1), pp. 64-80.

BILBAO-OSORIO B. y RODRIGUEZ-POSE A. (2004). "From R\&D to Innovation and Economic Growth in the EU". Growth and Change, 35(4), pp. 434-455.

BRESCHI, S. y MALERBA, F. (1997). "Sectoral Innovation Systems: Technological Regimes, Schumpeterian Dynamics, and Spatial Boundaries". En Edquist, C. (ed.): Systems of Innovation. Technologies, Institutions and Organizations (pp. 130-156). London: Pinter Publishers.

CHEN, K. y GUAN, J. (2011). "Mapping the Innovation Production Process from Accumulative Advantage to Economic Outcomes: A Path Modeling Approach". Technovation, 31(7), pp. 336-346.

DOSI, G.; PAVITT, K. y SOETE, L. (1990). The Economics of Technical Change and International Trade. Hemel Hampstead: Prentice Hall.

EUROPEAN COMMISSION (2009). Regional Innovation Scoreboard (RIS). Enterprise \& Industry Magazine. Inno Europe Paper $\mathrm{n}^{\circ} 14$ European Communities.

EUROPEAN COMMISSION (2010). Europe 2020. A strategy for Smart, Sustainable and Inclusive Growth. Communication from the Commission. European Union.

EUROPEAN COMMISSION (2012a). Regional Innovation Scoreboard (RIS). DirectorateGeneral for Enterprise and Industry. European Union.

EUROPEAN COMMISSION (2012b). Regional Innovation Monitor (RIM). Regional Innovation Report: Region of Andalusia. Directorate-General for Enterprise and Industry. European Union.

FORSMAN, H. (2011). "Innovation Capacity and Innovation Development in Small Enterprises. A Comparison Between the Manufacturing and Service Sectors". Research Policy, 40(5), pp. 739-750.

FREEMAN, C. (1987). Technology and Economic Performance: Lessons from Japan. London: Pinter Publishers. 
FRITSCH, M. (2000). "Interregional Differences in R\&D Activities: an Empirical investigation". European Planning Studies, 8(4), pp. 409-427.

FRITSCH, M. y ANDREAS, S. (2005). "Regionalization of Innovation Policy-Introduction to the Special Issue". Research Policy, 34(8), pp. 1123-1127.

HERVÁS-OLIVER J.L.; SEMPERE-RIPOLL F. y BORONAT-MOLL C. (2014). "Process Innovation Strategy in SMEs, Organizational Innovation and Performance: A Misleading Debate?". Small Business Economics, 43(4), pp. 873-886.

HOWELLS, J. (2005). "Innovation and Regional Economic Development: A Matter of Perspective?". Research Policy, 34(8), pp. 1220-1234.

HULL, C.E. y COVIN, J.G. (2010). "Learning Capability, Technological Parity, and Innovation Mode Use". Journal of Product Innovation Management, 27(1), pp. 97-114.

HURLEY, R.F. y HULT, G.T. (1998). "Innovation, Market Orientation, and Organizational Learning: An integration and Empirical Examination". Journal of Marketing, 62 (3), $42-$ 54.

KAUFMANN, A. y TÖDTLING, F. (2001). "Science-Industry Interaction in the Process of Innovation: The Importance of Boundary-Crossing Between Systems". Research Policy, 30(5), pp. 791-804.

KAUFMANN, A. y TÖDTLING, F. (2002). "How Effective is Innovation Support for SMEs? An Analysis of the Region of Upper Austria". Technovation, 22(3), pp. 147-159.

KESSLER, E.H.; BIERLY, P.E. y GOPALAKRISHNAN, S. (2000). "Internal vs. External Learning in New Product Development: Effects on Speed, Costs and Competitive Advantage". R\&D Management, 30(3), pp. 213-223.

KLEER, R. (2010). "Government R\&D Subsidies as a Signal for Private Investors". Research Policy, 39(10), pp. 1361-1374.

KOSCHATZKY, K. (1998). "Firm Innovation and Region: The Role of Space in Innovation Processes". International Journal Innovation Management, 2(4), pp. 383408.

MARTíNEZ-ROMÁN, J.A.; GAMERO, J. y TAMAYO, J.A. (2011). "Analysis of innovation in SMEs Using an Innovative Capability-based Non-linear Model: A Study in the Province of Seville (Spain)". Technovation, 31(9), pp. 459-475.

MARTíNEZ-ROMÁN, J.A.; TAMAYO, J.A.; GAMERO, J. y ROMERO, J.E. (2015). "Innovativeness and Business Performances in Tourism SMEs". Annals of Tourism Research, 54, pp. 118-135.

MARTÍNEZ-ROMÁN, J.A.; TAMAYO, J.A. y GAMERO, J. (2017). "Innovativeness and its Influence on Growth and Market Extension in Construction Firms in the Andalusian Region". Journal of Engineering and Technology Management, 43, pp. 19-33.

MENGUC, B. y AUH, S. (2010). "Development and Return on Execution of Product Innovation Capabilities: The Role of Organizational Structure". Industrial Marketing Management, 39(5), pp. 820-831.

MONTALVO, C. (2006). "What Triggers Change and Innovation?". Technovation, 26(3), pp. 312-323.

OLIVEIRA, T. y MARTINS, M.F. (2010). "Understanding e-Business Adoption Across Industries in European Countries". Industrial Management \& Data Systems, 110(8-9), pp. 1337-1354.

PORTER, M.E. (1991). La Ventaja Competitiva de las Naciones. Barcelona: Plaza \& Janés. 
RADAS, S. y BOZIC, L. (2009). "The Antecedents of SMEs Innovativeness in an Emerging Transition Economy". Technovation, 29(6-7), pp. 438-450.

ROMIJN, H. y ALBALADEJO, M. (2002). "Determinants of Innovation Capability in Small Electronics and Software Firms in Southeast England". Research Policy, 31(7), pp. 1053-1067.

RONDÉ, P. y HUSSLER, C. (2005). "Innovation in Regions: What does Really Matter?". Research Policy, 34(8), pp. 1150-1172.

RUSSELL, R.D. (1990). "Innovations in Organizations: Toward and Integrated Model". Review of Business, 12(2), pp. 19-25.

STERNBERG, R. y ARNDT, O. (2001). "The Firm or the Region: What Determines the innovation Behavior of European Firms?". Economic Geography, 77(4), pp. 345-363.

TÖDTLING, F. y TRIPPL, M. (2005). "One Size Fits All? Towards a Differentiated Regional Innovation Policy Approach". Research Policy, 34(8), pp. 1203-1219.

UYARRA, E. (2010). "What is Evolutionary About "Regional Systems of Innovation"? Implications for regional policy". Journal of Evolutionary Economics, 20(1), pp. 115-137.

VEGA-JURADO, J.; GUTIÉRREZ-GARCÍA, A.; FERNÁNDEZ-DE-LUCIO, I. у MANJARRÉS-HENRÍQUEZ, L. (2008). "The Effect of External and Internal Factors on Firms' Product Innovation". Research Policy, 37(4), pp. 616-632.

YAM, R.C.M.; LO, W.; TANG, E.P.Y. y LAU, A.K.W. (2011). "Analysis of Sources of Innovation, Technological Innovation Capabilities, and Performance: An Empirical Study of Hong Kong Manufacturing Industries". Research Policy, 40(3), pp. 391-402. 


\section{Apéndice}

\section{Tabla 8}

Tipología de empresa innovadora vs. no innovadora

\begin{tabular}{|c|c|c|c|c|c|}
\hline Tipos & Categorías & INNOV & IADORAS & NO INN & OVADORAS \\
\hline \multirow{6}{*}{ 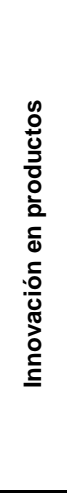 } & $\begin{array}{l}\text { Capacidad } \\
\text { innovadora }\end{array}$ & $\begin{array}{l}\text { Existencia de } \\
\text { patentes y otra } \\
\text { propiedad industrial }\end{array}$ & $\begin{array}{l}\text { Más asistencia a ferias } \\
\text { y congresos de no } \\
\text { directivos }\end{array}$ & $\begin{array}{l}\text { Menos asistencia a } \\
\text { ferias y congresos de } \\
\text { no directivos }\end{array}$ & $\begin{array}{l}\text { No existen patentes y otra } \\
\text { propiedad industrial }\end{array}$ \\
\hline & & $\begin{array}{l}\text { Mayor esfuerzo en } \\
\text { I+D }\end{array}$ & $\begin{array}{l}\text { Mayor } \% \text { de } \\
\text { universitarios directivos }\end{array}$ & $\begin{array}{l}\text { Menor \% de } \\
\text { universitarios } \\
\text { directivos }\end{array}$ & Menor esfuerzo en I+D \\
\hline & & $\begin{array}{l}\text { Más valoración de la } \\
\text { creatividad }\end{array}$ & & & $\begin{array}{l}\text { Menos valoración de la } \\
\text { creatividad }\end{array}$ \\
\hline & $\begin{array}{l}\text { Factores } \\
\text { contextuales }\end{array}$ & $\begin{array}{l}\text { Mayor tamaño ( } \mathrm{n}^{\circ} \\
\text { trabajadores) }\end{array}$ & & & $\begin{array}{l}\text { Menor tamaño }\left(n^{\circ}\right. \\
\text { trabajadores) }\end{array}$ \\
\hline & Entorno & $\begin{array}{l}\text { Mayor } n^{0} \text { de } \\
\text { deducciones I+D en } \\
\text { IS }\end{array}$ & & & $\begin{array}{l}\text { Menor } n^{\circ} \text { de deducciones } \\
\text { I+D en IS }\end{array}$ \\
\hline & & $\begin{array}{l}\text { Mayor utilización del } \\
\text { apoyo institucional a } \\
\text { la exportación }\end{array}$ & & & $\begin{array}{l}\text { Menor utilización del } \\
\text { apoyo institucional a la } \\
\text { exportación }\end{array}$ \\
\hline \multirow{7}{*}{ 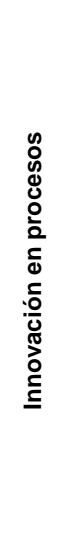 } & $\begin{array}{l}\text { Capacidad } \\
\text { innovadora }\end{array}$ & $\begin{array}{l}\text { Mayor esfuerzo en } \\
\text { I+D }\end{array}$ & $\begin{array}{l}\text { Más asistencia a ferias } \\
\text { y congresos de } \\
\text { directivos }\end{array}$ & $\begin{array}{l}\text { Menos asistencia a } \\
\text { ferias y congresos de } \\
\text { directivos }\end{array}$ & Menor esfuerzo en I+D \\
\hline & & $\begin{array}{l}\text { Existencia de grupos } \\
\text { de trabajo } \\
\text { permanentes }\end{array}$ & & & $\begin{array}{l}\text { No existen grupos de } \\
\text { trabajo permanentes }\end{array}$ \\
\hline & & & $\begin{array}{l}\text { Mayor nivel de } \\
\text { supervisión y control }\end{array}$ & $\begin{array}{l}\text { Menor nivel de } \\
\text { supervisión y control }\end{array}$ & \\
\hline & & & $\begin{array}{l}\text { Mayor orientación hacia } \\
\text { la estrategia } \\
\text { competitiva basada } \\
\text { diferenciación }\end{array}$ & $\begin{array}{l}\text { Mayor orientación } \\
\text { hacia la estrategia } \\
\text { competitiva basada en } \\
\text { costes }\end{array}$ & \\
\hline & $\begin{array}{l}\text { Factores } \\
\text { contextuales }\end{array}$ & $\begin{array}{l}\text { Más cooperación } \\
\text { con clientes }\end{array}$ & $\begin{array}{l}\text { Más cooperación con } \\
\text { distribuidores }\end{array}$ & $\begin{array}{l}\text { Menos cooperac. con } \\
\text { distribuidores }\end{array}$ & $\begin{array}{l}\text { Menos cooperación con } \\
\text { clientes }\end{array}$ \\
\hline & & & $\begin{array}{l}\text { Más cooperación con } \\
\text { competidores }\end{array}$ & $\begin{array}{l}\text { Menos cooperac. con } \\
\text { competidores }\end{array}$ & \\
\hline & Entorno & $\begin{array}{l}\text { Mayor importancia } \\
\text { deducción por I+D }\end{array}$ & & & $\begin{array}{l}\text { Mayor importancia deducc. } \\
\text { por I+D }\end{array}$ \\
\hline
\end{tabular}

Fuente: Elaboración propia. 
